\title{
A multi-dimensional functional equation having cubic forms as solutions
}

\author{
Won-Gil Park ${ }^{a}$, Jae-Hyeong Bae ${ }^{b, *}$ \\ ${ }^{a}$ Department of Mathematics Education, College of Education, Mokwon University, Daejeon 35349, Republic of Korea. \\ ${ }^{b}$ Humanitas College, Kyung Hee University, Yongin 17104, Republic of Korea.
}

Communicated by C. Park

\section{Abstract}

In this paper, we obtain some results on the $m$-variable cubic functional equation

$$
\begin{aligned}
& f\left(2 x_{1}+y_{1}, \cdots, 2 x_{m}+y_{m}\right)+f\left(2 x_{1}-y_{1}, \cdots, 2 x_{m}-y_{m}\right) \\
& \quad=2 f\left(x_{1}+y_{1}, \cdots, x_{m}+y_{m}\right)+2 f\left(x_{1}-y_{1}, \cdots, x_{m}-y_{m}\right)+12 f\left(x_{1}, \cdots, x_{m}\right) .
\end{aligned}
$$

The cubic form $f\left(x_{1}, \cdots, x_{m}\right)=\sum_{1 \leq i \leq j \leq k \leq m} a_{i j k} x_{i} x_{j} x_{k}$ is a solution of the above functional equation. (C)2016 all rights reserved.

Keywords: Cubic form, solution, stability.

2010 MSC: 39B52, 39B82.

\section{Introduction}

In this paper, let $X$ and $Y$ be real vector spaces. A mapping $f$ is called a cubic form (homogeneous polynomial of degree 3$)$ if there exists $a_{i j k} \in \mathbb{R}(1 \leq i \leq j \leq k \leq m)$ such that

$$
f\left(x_{1}, \cdots, x_{m}\right)=\sum_{1 \leq i \leq j \leq k \leq m} a_{i j k} x_{i} x_{j} x_{k}
$$

for all $x_{1}, \cdots, x_{m} \in X$. For a mapping $f: X^{m} \rightarrow Y$, consider the $m$-variable cubic functional equation:

$$
\begin{aligned}
& f\left(2 x_{1}+y_{1}, \cdots, 2 x_{m}+y_{m}\right)+f\left(2 x_{1}-y_{1}, \cdots, 2 x_{m}-y_{m}\right) \\
& \quad=2 f\left(x_{1}+y_{1}, \cdots, x_{m}+y_{m}\right)+2 f\left(x_{1}-y_{1}, \cdots, x_{m}-y_{m}\right)+12 f\left(x_{1}, \cdots, x_{m}\right) .
\end{aligned}
$$

\footnotetext{
* Corresponding author

Email addresses: wgpark@mokwon.ac.kr (Won-Gil Park), jhbae@khu.ac.kr (Jae-Hyeong Bae)
} 
When $X=Y=\mathbb{R}$, the cubic form $f: \mathbb{R}^{m} \rightarrow \mathbb{R}$ given by (1.1) is a solution of 1.2 .

For a mapping $g: X \rightarrow Y$, consider the cubic functional equation:

$$
g(2 x+y)+g(2 x-y)=2 g(x+y)+2 g(x-y)+12 g(x) .
$$

In 2002, Jun and Kim [4] solved the equation (1.3). Later, many different cubic functional equations were solved by numerous authors $([2 / 5])$.

In 2008, the authors [1] investigated the solution and stability of 1.2 for the case $m=2$. In this paper, we investigate the relation between $(1.2)$ and $(1.3)$ and some sufficient conditions that satisfy the equation (1.2), and prove the generalized Hyers-Ulam stability of (1.2).

\section{Results}

The $m$-variable cubic functional equation $(1.2)$ induces the cubic functional equation $(1.3)$ as follows.

Theorem 2.1. Let $f: X^{m} \rightarrow Y$ be a mapping satisfying 1.2 and let $g: X \rightarrow Y$ be the mapping given by

$$
g(x):=f(x, \cdots, x)
$$

for all $x \in X$, then $g$ satisfies 1.3 .

Proof. By (1.2) and 2.1), we have

$$
\begin{aligned}
g(2 x+y)+g(2 x-y) & =f(2 x+y, \cdots, 2 x+y)+f(2 x-y, \cdots, 2 x-y) \\
& =2 f(x+y, \cdots, x+y)+2 f(x-y, \cdots, x-y)+12 f(x, \cdots, x) \\
& =2 g(x+y)+2 g(x-y)+12 g(x)
\end{aligned}
$$

for all $x, y \in X$.

The cubic functional equation (1.3) induces the $m$-variable cubic functional equation (1.2) with an additional condition.

Theorem 2.2. Let $a_{i j k} \in \mathbb{R}(1 \leq i \leq j \leq k \leq m)$ and $g: X \rightarrow Y$ be a mapping satisfying (1.3). If $f: X^{m} \rightarrow Y$ is the mapping given by

$$
\begin{aligned}
f\left(x_{1}, \cdots, x_{m}\right):= & \sum_{i=1}^{m} a_{i i i} g\left(x_{i}\right)+\frac{1}{24} \sum_{1 \leq i<j \leq m}\left(a_{i i j}\left[g\left(2 x_{i}+x_{j}\right)-g\left(2 x_{i}-x_{j}\right)-2 g\left(x_{j}\right)\right]\right. \\
& \left.+a_{i j j}\left[g\left(x_{i}+2 x_{j}\right)+g\left(x_{i}-2 x_{j}\right)-2 g\left(x_{i}\right)\right]\right) \\
& +\frac{1}{6} \sum_{1 \leq i<j<k \leq m} a_{i j k}\left[7 g\left(x_{i}+x_{j}+x_{k}\right)+2 g\left(x_{i}\right)+2 g\left(x_{j}\right)+2 g\left(x_{k}\right)\right. \\
& \left.-g\left(2 x_{i}+x_{j}+x_{k}\right)-g\left(x_{i}+2 x_{j}+x_{k}\right)-g\left(x_{i}+x_{j}+2 x_{k}\right)\right]
\end{aligned}
$$

for all $x_{1}, \cdots, x_{m} \in X$, then $f$ satisfies 1.2 .

Furthermore, 2.1) holds if $\sum_{i=1}^{m} a_{i i i}+\sum_{1 \leq i<j \leq m}\left(a_{i i j}+a_{i j j}\right)+\frac{1}{2} \sum_{1 \leq i<j<k \leq m} a_{i j k}=1$.

Proof. By (1.3) and 2.2), we obtain

$$
\begin{aligned}
& f\left(2 x_{1}+y_{1}, \cdots, 2 x_{m}+y_{m}\right)+f\left(2 x_{1}-y_{1}, \cdots, 2 x_{m}-y_{m}\right) \\
& \quad=\sum_{i=1}^{m} a_{i i i}\left[g\left(2 x_{i}+y_{i}\right)+g\left(2 x_{i}-y_{i}\right)\right]
\end{aligned}
$$




$$
\begin{aligned}
& +\frac{1}{24} \sum_{1 \leq i<j \leq m}\left(a _ { i i j } \left[g\left(4 x_{i}+2 x_{j}+2 y_{i}+y_{j}\right)-g\left(4 x_{i}-2 x_{j}+2 y_{i}-y_{j}\right)\right.\right. \\
& \left.-2 g\left(2 x_{j}+y_{j}\right)+g\left(4 x_{i}+2 x_{j}-2 y_{i}-y_{j}\right)-g\left(4 x_{i}-2 x_{j}-2 y_{i}+y_{j}\right)-2 g\left(2 x_{j}-y_{j}\right)\right] \\
& +a_{i j j}\left[g\left(2 x_{i}+4 x_{j}+y_{i}+2 y_{j}\right)+g\left(2 x_{i}-4 x_{j}+y_{i}-2 y_{j}\right)\right. \\
& \left.\left.-2 g\left(2 x_{i}+y_{i}\right)+g\left(2 x_{i}+4 x_{j}-y_{i}-2 y_{j}\right)+g\left(2 x_{i}-4 x_{j}-y_{i}+2 y_{j}\right)-2 g\left(2 x_{i}-y_{i}\right)\right]\right) \\
& +\frac{1}{6} \sum_{1 \leq i<j<k \leq m} a_{i j k}\left[7 g\left(2 x_{i}+2 x_{j}+2 x_{k}+y_{i}+y_{j}+y_{k}\right)+2 g\left(2 x_{i}+y_{i}\right)\right. \\
& +2 g\left(2 x_{j}+y_{j}\right)+2 g\left(2 x_{k}+y_{k}\right)-g\left(4 x_{i}+2 x_{j}+2 x_{k}+2 y_{i}+y_{j}+y_{k}\right) \\
& -g\left(2 x_{i}+4 x_{j}+2 x_{k}+y_{i}+2 y_{j}+y_{k}\right)-g\left(2 x_{i}+2 x_{j}+4 x_{k}+y_{i}+y_{j}+2 y_{k}\right) \\
& +7 g\left(2 x_{i}+2 x_{j}+2 x_{k}-y_{i}-y_{j}-y_{k}\right)+2 g\left(2 x_{i}-y_{i}\right) \\
& +2 g\left(2 x_{j}-y_{j}\right)+2 g\left(2 x_{k}-y_{k}\right)-g\left(4 x_{i}+2 x_{j}+2 x_{k}-2 y_{i}-y_{j}-y_{k}\right) \\
& \left.-g\left(2 x_{i}+4 x_{j}+2 x_{k}-y_{i}-2 y_{j}-y_{k}\right)-g\left(2 x_{i}+2 x_{j}+4 x_{k}-y_{i}-y_{j}-2 y_{k}\right)\right] \\
& =\left(\sum_{i=1}^{m} a_{i i i}-\frac{1}{12} \sum_{1 \leq i<j \leq m} a_{i j j}+\frac{1}{3} \sum_{1 \leq i<j<k \leq m} a_{i j k}\right)\left[g\left(2 x_{i}+y_{i}\right)+g\left(2 x_{i}-y_{i}\right)\right] \\
& -\left(\frac{1}{12} \sum_{1 \leq i<j \leq m} a_{i i j}-\frac{1}{3} \sum_{1 \leq i<j<k \leq m} a_{i j k}\right)\left[g\left(2 x_{j}+y_{j}\right)+g\left(2 x_{j}-y_{j}\right)\right] \\
& +\frac{1}{3} \sum_{1 \leq i<j<k \leq m} a_{i j k}\left[g\left(2 x_{k}+y_{k}\right)+g\left(2 x_{k}-y_{k}\right)\right] \\
& +\frac{1}{24} \sum_{1 \leq i<j \leq m}\left[a _ { i i j } \left(g\left[2\left(2 x_{i}+x_{j}\right)+\left(2 y_{i}+y_{j}\right)\right]+g\left[2\left(2 x_{i}+x_{j}\right)-\left(2 y_{i}+y_{j}\right)\right]\right.\right. \\
& \left.-g\left[2\left(2 x_{i}-x_{j}\right)+\left(2 y_{i}-y_{j}\right)\right]-g\left[2\left(2 x_{i}-x_{j}\right)-\left(2 y_{i}-y_{j}\right)\right]\right) \\
& +a_{i j j}\left(g\left[2\left(x_{i}+2 x_{j}\right)+\left(y_{i}+2 y_{j}\right)\right]+g\left[2\left(x_{i}-2 x_{j}\right)+\left(y_{i}-2 y_{j}\right)\right]\right. \\
& \left.\left.+g\left[2\left(x_{i}+2 x_{j}\right)-\left(y_{i}+2 y_{j}\right)\right]+g\left[2\left(x_{i}-2 x_{j}\right)-\left(y_{i}-2 y_{j}\right)\right]\right)\right]+\frac{1}{6} \sum_{1 \leq i<j<k \leq m} a_{i j k} \\
& \left(7 g\left[2\left(x_{i}+x_{j}+x_{k}\right)+\left(y_{i}+y_{j}+y_{k}\right)\right]+7 g\left[2\left(x_{i}+x_{j}+x_{k}\right)-\left(y_{i}+y_{j}+y_{k}\right)\right]\right. \\
& -g\left[2\left(2 x_{i}+x_{j}+x_{k}\right)+\left(2 y_{i}+y_{j}+y_{k}\right)\right]-g\left[2\left(2 x_{i}+x_{j}+x_{k}\right)-\left(2 y_{i}+y_{j}+y_{k}\right)\right] \\
& -g\left[2\left(x_{i}+2 x_{j}+x_{k}\right)+\left(y_{i}+2 y_{j}+y_{k}\right)\right]-g\left[2\left(x_{i}+2 x_{j}+x_{k}\right)-\left(y_{i}+2 y_{j}+y_{k}\right)\right] \\
& \left.-g\left[2\left(x_{i}+x_{j}+2 x_{k}\right)+\left(y_{i}+y_{j}+2 y_{k}\right)\right]-g\left[2\left(x_{i}+x_{j}+2 x_{k}\right)-\left(y_{i}+y_{j}+2 y_{k}\right)\right]\right) \\
& =\left(2 \sum_{i=1}^{m} a_{i i i}-\frac{1}{6} \sum_{1 \leq i<j \leq m} a_{i j j}+\frac{2}{3} \sum_{1 \leq i<j<k \leq m} a_{i j k}\right)\left[g\left(x_{i}+y_{i}\right)+g\left(x_{i}-y_{i}\right)+6 g\left(x_{i}\right)\right] \\
& -\left(\frac{1}{6} \sum_{1 \leq i<j \leq m} a_{i i j}-\frac{2}{3} \sum_{1 \leq i<j<k \leq m} a_{i j k}\right)\left[g\left(x_{j}+y_{j}\right)+g\left(x_{j}-y_{j}\right)+6 g\left(x_{j}\right)\right] \\
& +\frac{2}{3} \sum_{1 \leq i<j<k \leq m} a_{i j k}\left[g\left(x_{k}+y_{k}\right)+g\left(x_{k}-y_{k}\right)+6 g\left(x_{k}\right)\right]
\end{aligned}
$$




$$
\begin{aligned}
& +\frac{1}{12} \sum_{1 \leq i<j \leq m}\left[a _ { i i j } \left(g\left[\left(2 x_{i}+x_{j}\right)+\left(2 y_{i}+y_{j}\right)\right]+g\left[\left(2 x_{i}+x_{j}\right)-\left(2 y_{i}+y_{j}\right)\right]\right.\right. \\
& \left.+6 g\left(2 x_{i}+x_{j}\right)-g\left[\left(2 x_{i}-x_{j}\right)+\left(2 y_{i}-y_{j}\right)\right]-g\left[\left(2 x_{i}-x_{j}\right)-\left(2 y_{i}-y_{j}\right)\right]-6 g\left(2 x_{i}-x_{j}\right)\right) \\
& +a_{i j j}\left(g\left[\left(x_{i}+2 x_{j}\right)+\left(y_{i}+2 y_{j}\right)\right]+g\left[\left(x_{i}+2 x_{j}\right)-\left(y_{i}+2 y_{j}\right)\right]+6 g\left(x_{i}+2 x_{j}\right)\right. \\
& \left.\left.+g\left[\left(x_{i}-2 x_{j}\right)+\left(y_{i}-2 y_{j}\right)\right]+g\left[\left(x_{i}-2 x_{j}\right)-\left(y_{i}-2 y_{j}\right)\right]+6 g\left(x_{i}-2 x_{j}\right)\right)\right] \\
& +\frac{1}{3} \sum_{1 \leq i<j<k \leq m} a_{i j k}\left(7 g\left[\left(x_{i}+x_{j}+x_{k}\right)+\left(y_{i}+y_{j}+y_{k}\right)\right]+7 g\left[\left(x_{i}+x_{j}+x_{k}\right)-\left(y_{i}+y_{j}+y_{k}\right)\right]\right. \\
& +42 g\left(x_{i}+x_{j}+x_{k}\right)-g\left[\left(2 x_{i}+x_{j}+x_{k}\right)+\left(2 y_{i}+y_{j}+y_{k}\right)\right] \\
& -g\left[\left(2 x_{i}+x_{j}+x_{k}\right)-\left(2 y_{i}+y_{j}+y_{k}\right)\right]-6 g\left(2 x_{i}+x_{j}+x_{k}\right) \\
& -g\left[\left(x_{i}+2 x_{j}+x_{k}\right)+\left(y_{i}+2 y_{j}+y_{k}\right)\right]-g\left[\left(x_{i}+2 x_{j}+x_{k}\right)-\left(y_{i}+2 y_{j}+y_{k}\right)\right] \\
& -6 g\left(x_{i}+2 x_{j}+x_{k}\right)-g\left[\left(x_{i}+x_{j}+2 x_{k}\right)+\left(y_{i}+y_{j}+2 y_{k}\right)\right] \\
& \left.-g\left[\left(x_{i}+x_{j}+2 x_{k}\right)-\left(y_{i}+y_{j}+2 y_{k}\right)\right]-6 g\left(x_{i}+x_{j}+2 x_{k}\right)\right) \\
& =2 \sum_{i=1}^{m} a_{i i i}\left[g\left(x_{i}+y_{i}\right)+g\left(x_{i}-y_{i}\right)+6 g\left(x_{i}\right)\right] \\
& +\frac{1}{12} \sum_{1 \leq i<j \leq m}\left(a _ { i i j } \left[g\left(2 x_{i}+x_{j}+2 y_{i}+y_{j}\right)-g\left(2 x_{i}-x_{j}+2 y_{i}-y_{j}\right)\right.\right. \\
& -2 g\left(x_{j}+y_{j}\right)+g\left(2 x_{i}+x_{j}-2 y_{i}-y_{j}\right)-g\left(2 x_{i}-x_{j}-2 y_{i}+y_{j}\right) \\
& \left.-2 g\left(x_{j}-y_{j}\right)+6 g\left(2 x_{i}+x_{j}\right)-6 g\left(2 x_{i}-x_{j}\right)-12 g\left(x_{j}\right)\right] \\
& +a_{i j j}\left[g\left(x_{i}+2 x_{j}+y_{i}+2 y_{j}\right)+g\left(x_{i}-2 x_{j}+y_{i}-2 y_{j}\right)\right. \\
& -2 g\left(x_{i}+y_{i}\right)+g\left(x_{i}+2 x_{j}-y_{i}-2 y_{j}\right)+g\left(x_{i}-2 x_{j}-y_{i}+2 y_{j}\right) \\
& \left.\left.-2 g\left(x_{i}-y_{i}\right)+6 g\left(x_{i}+2 x_{j}\right)+6 g\left(x_{i}-2 x_{j}\right)-12 g\left(x_{i}\right)\right]\right) \\
& +\frac{1}{3} \sum_{1 \leq i<j<k \leq m} a_{i j k}\left[7 g\left(x_{i}+x_{j}+x_{k}+y_{i}+y_{j}+y_{k}\right)+2 g\left(x_{i}+y_{i}\right)+2 g\left(x_{j}+y_{j}\right)+2 g\left(x_{k}+y_{k}\right)\right. \\
& -g\left(2 x_{i}+x_{j}+x_{k}+2 y_{i}+y_{j}+y_{k}\right)-g\left(x_{i}+2 x_{j}+x_{k}+y_{i}+2 y_{j}+y_{k}\right) \\
& -g\left(x_{i}+x_{j}+2 x_{k}+y_{i}+y_{j}+2 y_{k}\right)+7 g\left(x_{i}+x_{j}+x_{k}-y_{i}-y_{j}-y_{k}\right) \\
& +2 g\left(x_{i}-y_{i}\right)+2 g\left(x_{j}-y_{j}\right)+2 g\left(x_{k}-y_{k}\right) \\
& -g\left(2 x_{i}+x_{j}+x_{k}-2 y_{i}-y_{j}-y_{k}\right)-g\left(x_{i}+2 x_{j}+x_{k}-y_{i}-2 y_{j}-y_{k}\right) \\
& -g\left(x_{i}+x_{j}+2 x_{k}-y_{i}-y_{j}-2 y_{k}\right)+42 g\left(x_{i}+x_{j}+x_{k}\right)+12 g\left(x_{i}\right) \\
& \left.+12 g\left(x_{j}\right)+12 g\left(x_{k}\right)-6 g\left(2 x_{i}+x_{j}+x_{k}\right)-6 g\left(x_{i}+2 x_{j}+x_{k}\right)-6 g\left(x_{i}+x_{j}+2 x_{k}\right)\right] \\
& =2 f\left(x_{1}+y_{1}, \cdots, x_{m}+y_{m}\right)+2 f\left(x_{1}-y_{1}, \cdots, x_{m}-y_{m}\right)+12 f\left(x_{1}, \cdots, x_{m}\right)
\end{aligned}
$$

for all $x_{1}, \cdots, x_{m}, y_{1}, \cdots, y_{m} \in X$. Letting $x=y=0$ in $(1.3)$, we get $g(0)=0$. Putting $y=0$ in $(1.3)$, we obtain $g(2 x)=8 g(x)$ for all $x \in X$. And putting $y=x$ in $(1.3)$, we infer $g(3 x)=27 g(x)$ for all $x \in X$. Setting $x=0$ in 1.3 , we have that $g$ is an odd mapping. If $\sum_{i=1}^{m} a_{i i i}+\sum_{1 \leq i<j \leq m}\left(a_{i i j}+a_{i j j}\right)+\frac{1}{2} \sum_{1 \leq i<j<k \leq m} a_{i j k}=$ 
1 , by 2.2 , we find

$$
\begin{aligned}
f(x, \cdots, x)= & \sum_{i=1}^{m} a_{i i i} g(x)+\frac{1}{24} \sum_{1 \leq i<j \leq m}\left(a_{i i j}[g(3 x)-3 g(x)]+a_{i j j}[g(3 x)+g(-x)-2 g(x)]\right) \\
& +\frac{1}{6} \sum_{1 \leq i<j<k \leq m} a_{i j k}[7 g(3 x)+6 g(x)-3 g(4 x)] \\
= & \sum_{i=1}^{m} a_{i i i} g(x)+\sum_{1 \leq i<j \leq m}\left[a_{i i j} g(x)+a_{i j j} g(x)\right]+\frac{1}{2} \sum_{1 \leq i<j<k \leq m} a_{i j k} g(x) \\
= & {\left[\sum_{i=1}^{m} a_{i i i}+\sum_{1 \leq i<j \leq m}\left(a_{i i j}+a_{i j j}\right)+\frac{1}{2} \sum_{1 \leq i<j<k \leq m} a_{i j k}\right] g(x) } \\
= & g(x)
\end{aligned}
$$

for all $x \in X$.

A mapping $S: X^{3} \rightarrow Y$ is called symmetric if

$$
S(x, y, z)=S(x, z, y)=S(y, x, z)=S(y, z, x)=S(z, x, y)=S(z, y, x)
$$

for all $x, y, z \in X$.

In the following theorem, we find out some sufficient conditions that satisfy the equation $(1.2)$.

Theorem 2.3. A mapping $f: X^{m} \rightarrow Y$ satisfies (1.2) if there exist symmetric multi-additive mappings $S_{1}, \cdots, S_{m}: X^{3} \rightarrow Y$ and multi-additive mappings $L_{i j}, N_{i j}: X^{3} \rightarrow Y(1 \leq i<j \leq m), M_{i j k}: X^{3} \rightarrow Y(1 \leq$ $i<j<k \leq m)$ such that

$$
f\left(x_{1}, \cdots, x_{m}\right)=\sum_{i=1}^{m} S_{i}\left(x_{i}, x_{i}, x_{i}\right)+\sum_{1 \leq i<j \leq m}\left[L_{i j}\left(x_{i}, x_{i}, x_{j}\right)+N_{i j}\left(x_{i}, x_{j}, x_{j}\right)\right]+\sum_{1 \leq i<j<k \leq m} M_{i j k}\left(x_{i}, x_{j}, x_{k}\right),
$$

$L_{i j}(x, y, z)=L_{i j}(y, x, z)$ and $N_{i j}(x, y, z)=N_{i j}(x, z, y)$ for all $x, y, z, x_{1}, \cdots, x_{m} \in X$.

Proof. We assume that there exist symmetric multi-additive mappings $S_{1}, \cdots, S_{m}: X^{3} \rightarrow Y$ and multiadditive mappings $L_{i j}, N_{i j}: X^{3} \rightarrow Y(1 \leq i<j \leq m), M_{i j k}: X^{3} \rightarrow Y(1 \leq i<j<k \leq m)$ such that

$$
f\left(x_{1}, \cdots, x_{m}\right)=\sum_{i=1}^{m} S_{i}\left(x_{i}, x_{i}, x_{i}\right)+\sum_{1 \leq i<j \leq m}\left[L_{i j}\left(x_{j}, x_{i}, x_{i}\right)+N_{i j}\left(x_{i}, x_{j}, x_{j}\right)\right]+\sum_{1 \leq i<j<k \leq m} M_{i j k}\left(x_{i}, x_{j}, x_{k}\right),
$$

$L_{i j}(x, y, z)=L_{i j}(y, x, z)$ and $N_{i j}(x, y, z)=N_{i j}(x, z, y)$ for all $x, y, z, x_{1}, \cdots, x_{m} \in X$. Since $M_{i j}(1 \leq i<$ $j \leq m)$ are multi-additive and $S_{1}, \cdots, S_{m}$ are symmetric multi-additive,

$$
\begin{aligned}
f( & \left.2 x_{1}+y_{1}, \cdots, 2 x_{m}+y_{m}\right)+f\left(2 x_{1}-y_{1}, \cdots, 2 x_{m}-y_{m}\right) \\
= & \sum_{i=1}^{m} S_{i}\left(2 x_{i}+y_{i}, 2 x_{i}+y_{i}, 2 x_{i}+y_{i}\right) \\
& +\sum_{1 \leq i<j \leq m}\left[L_{i j}\left(2 x_{j}+y_{j}, 2 x_{i}+y_{i}, 2 x_{i}+y_{i}\right)+N_{i j}\left(2 x_{i}+y_{i}, 2 x_{j}+y_{j}, 2 x_{j}+y_{j}\right)\right] \\
& +\sum_{1 \leq i<j<k \leq m} M_{i j k}\left(2 x_{i}+y_{i}, 2 x_{j}+y_{j}, 2 x_{k}+y_{k}\right)+\sum_{i=1}^{m} S_{i}\left(2 x_{i}-y_{i}, 2 x_{i}-y_{i}, 2 x_{i}-y_{i}\right)
\end{aligned}
$$




$$
\begin{aligned}
& +\sum_{1 \leq i<j \leq m}\left[L_{i j}\left(2 x_{j}-y_{j}, 2 x_{i}-y_{i}, 2 x_{i}-y_{i}\right)+N_{i j}\left(2 x_{i}-y_{i}, 2 x_{j}-y_{j}, 2 x_{j}-y_{j}\right)\right] \\
& +\sum_{1 \leq i<j<k \leq m} M_{i j k}\left(2 x_{i}-y_{i}, 2 x_{j}-y_{j}, 2 x_{k}-y_{k}\right) \\
& =\sum_{i=1}^{m}\left[S_{i}\left(2 x_{i}+y_{i}, 2 x_{i}+y_{i}, 2 x_{i}+y_{i}\right)+S_{i}\left(2 x_{i}-y_{i}, 2 x_{i}-y_{i}, 2 x_{i}-y_{i}\right)\right] \\
& +4 \sum_{1 \leq i<j \leq m}\left[4 L_{i j}\left(x_{j}, x_{i}, x_{i}\right)+L_{i j}\left(x_{j}, y_{i}, y_{i}\right)+L_{i j}\left(y_{j}, x_{i}, y_{i}\right)+L_{i j}\left(y_{j}, y_{i}, x_{i}\right)\right. \\
& \left.+4 N_{i j}\left(x_{i}, x_{j}, x_{j}\right)+N_{i j}\left(x_{i}, y_{j}, y_{j}\right)+N_{i j}\left(y_{i}, x_{j}, y_{j}\right)+N_{i j}\left(y_{i}, y_{j}, x_{j}\right)\right] \\
& +4 \sum_{1 \leq i<j<k \leq m}\left[4 M_{i j k}\left(x_{i}, x_{j}, x_{k}\right)+M_{i j k}\left(x_{i}, y_{j}, y_{k}\right)+M_{i j k}\left(y_{i}, x_{j}, y_{k}\right)+M_{i j k}\left(y_{i}, y_{j}, x_{k}\right)\right] \\
& =2\left(\sum_{i=1}^{m} S_{i}\left(x_{i}+y_{i}, x_{i}+y_{i}, x_{i}+y_{i}\right)+\sum_{1 \leq i<j \leq m}\left[L_{i j}\left(x_{j}+y_{j}, x_{i}+y_{i}, x_{i}+y_{i}\right)\right.\right. \\
& \left.\left.+N_{i j}\left(x_{i}+y_{i}, x_{j}+y_{j}, x_{j}+y_{j}\right)\right]+\sum_{1 \leq i<j<k \leq m} M_{i j k}\left(x_{i}+y_{i}, x_{j}+y_{j}, x_{k}+y_{k}\right)\right) \\
& +2\left(\sum_{i=1}^{m} S_{i}\left(x_{i}-y_{i}, x_{i}-y_{i}, x_{i}-y_{i}\right)+\sum_{1 \leq i<j \leq m}\left[L_{i j}\left(x_{j}-y_{j}, x_{i}-y_{i}, x_{i}-y_{i}\right)\right.\right. \\
& \left.\left.+N_{i j}\left(x_{i}-y_{i}, x_{j}-y_{j}, x_{j}-y_{j}\right)\right]+\sum_{1 \leq i<j<k \leq m} M_{i j k}\left(x_{i}-y_{i}, x_{j}-y_{j}, x_{k}-y_{k}\right)\right) \\
& +12\left(\sum_{i=1}^{m} S_{i}\left(x_{i}, x_{i}, x_{i}\right)+\sum_{1 \leq i<j \leq m}\left[L_{i j}\left(x_{j}, x_{i}, x_{i}\right)+N_{i j}\left(x_{i}, x_{j}, x_{j}\right)\right]+\sum_{1 \leq i<j<k \leq m} M_{i j k}\left(x_{i}, x_{j}, x_{k}\right)\right) \\
& =2 f\left(x_{1}+y_{1}, \cdots, x_{m}+y_{m}\right)+2 f\left(x_{1}-y_{1}, \cdots, x_{m}-y_{m}\right)+12 f\left(x_{1}, \cdots, x_{m}\right)
\end{aligned}
$$

for all $x_{1}, \cdots, x_{m}, y_{1}, \cdots, y_{m} \in X$.

From now on, let $Y$ be complete and let $\varphi: X^{2 m} \rightarrow[0, \infty)$ be a function satisfying

$$
\tilde{\varphi}\left(x_{1}, \cdots, x_{m}, y_{1}, \cdots, y_{m}\right):=\sum_{j=0}^{\infty} \frac{1}{2 \cdot 8^{j+1}} \varphi\left(2^{j} x_{1}, \cdots, 2^{j} x_{m}, 2^{j} y_{1}, \cdots, 2^{j} y_{m}\right)<\infty
$$

for all $x_{1}, \cdots, x_{m}, y_{1}, \cdots, y_{m} \in X$.

Theorem 2.4. Let $f: X^{m} \rightarrow Y$ be a mapping such that

$$
\begin{aligned}
& \| f\left(2 x_{1}+y_{1}, \cdots, 2 x_{m}+y_{m}\right)+f\left(2 x_{1}-y_{1}, \cdots, 2 x_{m}-y_{m}\right) \\
& \quad-2 f\left(x_{1}+y_{1}, \cdots, x_{m}+y_{m}\right)-2 f\left(x_{1}-y_{1}, \cdots, x_{m}-y_{m}\right)-12 f\left(x_{1}, \cdots, x_{m}\right) \| \\
& \quad \leq \varphi\left(x_{1}, \cdots, x_{m}, y_{1}, \cdots, y_{m}\right)
\end{aligned}
$$

for all $x_{1}, \cdots, x_{m}, y_{1}, \cdots, y_{m} \in X$. Then there exists a unique mapping $F: X^{m} \rightarrow Y$ satisfying 1.2 such that

$$
\left\|f\left(x_{1}, \cdots, x_{m}\right)-F\left(x_{1}, \cdots, x_{m}\right)\right\| \leq \tilde{\varphi}\left(x_{1}, \cdots, x_{m}, 0, \cdots, 0\right)
$$

for all $x_{1}, \cdots, x_{m} \in X$, where the mapping $F$ is given by

$$
F\left(x_{1}, \cdots, x_{m}\right):=\lim _{j \rightarrow \infty} \frac{1}{8^{j}} f\left(2^{j} x_{1}, \cdots, 2^{j} x_{m}\right)
$$

for all $x_{1}, \cdots, x_{m} \in X$. 
Proof. Letting $y_{1}=\cdots=y_{m}=0$ in $(2.4)$, we have

$$
\left\|f\left(x_{1}, \cdots, x_{m}\right)-\frac{1}{8} f\left(2 x_{1}, \cdots, 2 x_{m}\right)\right\| \leq \frac{1}{16} \varphi\left(x_{1}, \cdots, x_{m}, 0, \cdots, 0\right)
$$

for all $x_{1}, \cdots, x_{m} \in X$. Thus we obtain

$$
\left\|\frac{1}{8^{j}} f\left(2^{j} x_{1}, \cdots, 2^{j} x_{m}\right)-\frac{1}{8^{j+1}} f\left(2^{j+1} x_{1}, \cdots, 2^{j+1} x_{m}\right)\right\| \leq \frac{1}{2 \cdot 8^{j+1}} \varphi\left(2^{j} x_{1}, \cdots, 2^{j} x_{m}, 0, \cdots, 0\right)
$$

for all $x_{1}, \cdots, x_{m} \in X$ and all $j$. For given integers $k, l(0 \leq k<l)$, we get

$$
\left\|\frac{1}{8^{k}} f\left(2^{k} x_{1}, \cdots, 2^{k} x_{m}\right)-\frac{1}{8^{l}} f\left(2^{l} x_{1}, \cdots, 2^{l} x_{m}\right)\right\| \leq \sum_{j=k}^{l-1} \frac{1}{2 \cdot 8^{j+1}} \varphi\left(2^{j} x_{1}, \cdots, 2^{j} x_{m}, 0, \cdots, 0\right)
$$

for all $x_{1}, \cdots, x_{m} \in X$. By $(2.6)$, the sequence $\left\{\frac{1}{8^{j}} f\left(2^{j} x_{1}, \cdots, 2^{j} x_{m}\right)\right\}$ is a Cauchy sequence for all $x_{1}, \cdots, x_{m} \in X$. Since $Y$ is complete, the sequence $\left\{\frac{1}{8^{j}} f\left(2^{j} x_{1}, \cdots, 2^{j} x_{m}\right)\right\}$ converges for all $x_{1}, \cdots, x_{m} \in X$. Define $F: X^{m} \rightarrow Y$ by

$$
F\left(x_{1}, \cdots, x_{m}\right):=\lim _{j \rightarrow \infty} \frac{1}{8^{j}} f\left(2^{j} x_{1}, \cdots, 2^{j} x_{m}\right)
$$

for all $x_{1}, \cdots, x_{m} \in X$. By (2.4), we have

$$
\begin{aligned}
\| \frac{1}{8^{j}} & {\left[f\left(2^{j}\left(2 x_{1}+y_{1}\right), \cdots, 2^{j}\left(2 x_{m}+y_{m}\right)\right)+f\left(2^{j}\left(2 x_{1}-y_{1}\right), \cdots, 2^{j}\left(2 x_{m}-y_{m}\right)\right)\right] } \\
& \quad-\frac{2}{8^{j}}\left[f\left(2^{j}\left(x_{1}+y_{1}\right), \cdots, 2^{j}\left(x_{m}+y_{m}\right)\right)-f\left(2^{j}\left(x_{1}-y_{1}\right), \cdots, 2^{j}\left(x_{m}-y_{m}\right)\right)\right]+6 f\left(x_{1}, \cdots, x_{m}\right) \| \\
\leq & \frac{1}{8^{j}} \varphi\left(2^{j} x_{1}, \cdots, 2^{j} x_{m}, 2^{j} y_{1}, \cdots, 2^{j} y_{m}\right)
\end{aligned}
$$

for all $x_{1}, \cdots, x_{m}, y_{1}, \cdots, y_{m} \in X$ and all $j$. Letting $j \rightarrow \infty$ and using (2.3), we see that $F$ satisfies $(1.2)$. Setting $k=0$ and taking $l \rightarrow \infty$ in (2.6), one can obtain the inequality (2.5). If $G: X^{m} \rightarrow Y$ is another mapping satisfying 1.2 and 2.5 , we obtain

$$
\begin{aligned}
\left\|F\left(x_{1}, \cdots, x_{m}\right)-G\left(x_{1}, \cdots, x_{m}\right)\right\|= & \frac{1}{8^{j}}\left\|F\left(2^{j} x_{1}, \cdots, 2^{j} x_{m}\right)-G\left(2^{j} x_{1}, \cdots, 2^{j} x_{m}\right)\right\| \\
\leq & \frac{1}{8^{j}}\left\|F\left(2^{j} x_{1}, \cdots, 2^{j} x_{m}\right)-f\left(2^{j} x_{1}, \cdots, 2^{j} x_{m}\right)\right\| \\
& +\frac{1}{8^{j}}\left\|f\left(2^{j} x_{1}, \cdots, 2^{j} x_{m}\right)-G\left(2^{j} x_{1}, \cdots, 2^{j} x_{m}\right)\right\| \\
\leq & \frac{2}{8^{j}} \tilde{\varphi}\left(2^{j} x_{1}, \cdots, 2^{j} x_{m}, 0, \cdots, 0\right) \rightarrow 0 \text { as } j \rightarrow \infty
\end{aligned}
$$

for all $x_{1}, \cdots, x_{m} \in X$. Hence the mapping $F$ is the unique mapping satisfying (1.2), as desired.

\section{References}

[1] J.-H. Bae, W.-G. Park, A functional equation on homogeneous polynomials, J. Korean Soc. Math. Educ. Ser. B, 15 (2008), 103-110. 1

[2] I.-S. Chang, Y.-S. Jung, Stability of a functional equation deriving from cubic and quadratic functions, J. Math. Anal. Appl., 283 (2003), 491-500. 1

[3] H.-Y. Chu, D.-S. Kang, On the stability of an n-dimensional cubic functional equation, J. Math. Anal. Appl., 325 (2007), 595-607.

[4] K.-W. Jun, H.-M. Kim, The generalized Hyers-Ulam-Rassias stability of a cubic functional equation, J. Math. Anal. Appl., 274 (2002), 867-878. 1

[5] K.-W. Jun, H.-M. Kim, I.-S. Chang, On the Hyers-Ulam stability of an Euler-Lagrange type cubic functional equation, J. Comput. Anal. Appl., 7 (2005), 21-33. 1 